\title{
Botulinum Toxin Can Abolish and/or Quiet Tinnitus Associated with Chronic Migraine: Serendipidous Observations
}

Danièle Ranoux ${ }^{1 *}$

Robert Aaron Levine ${ }^{2}$

\begin{abstract}
Introduction: It is well established that treatment of head and neck myofascial dysfunction can alleviate both tinnitus and chronic migraine $(\mathrm{CM})$. Onabotulinumtoxin $\mathrm{A}(\mathrm{OnaA})$ has become a standard treatment for $\mathrm{CM}$. In a recent systematic study a subject reported tinnitus improvement. This prompted a survey of the tinnitus in all study participants.

Methods: Fifty-seven patients with $\mathrm{CM}$ at a tertiary headache referral center received intramuscular Onabotulinumtoxin A (OnaA) injections into craniocervical muscles using the "follow-the-pain" protocol for headache. Effectiveness of OnaA was assessed by changes in (i) tinnitus loudness and (ii) number of headache days

Results: Of the five patients with pre-existing tinnitus OnaA abolished the tinnitus in two, including one whose tinnitus of ten years' duration resolved permanently with one treatment. The tinnitus loudness of the other four was attenuated between 70 to 100 percent for about three months, which paralleled their headaches response. All were women who had (i) a significant improvement of their $\mathrm{CM}$ and (ii) headaches located fronto-temporally. None of the CM non-responders reported tinnitus. Analysis of injection sites revealed that the temporalis muscle injections were likely accounting for the tinnitus attenuation.

Conclusion: Tinnitus associated with chronic migraine is abolished/quieted by intramuscular craniocervical Onabotulinumtoxin A injections. These results are consistent with the dorsal cochlear nucleus hypothesis for craniocervical somatic tinnitus, as well as the association between migraine and tinnitus. This serendipitous result warrants further study of botulinum toxin for tinnitus.
\end{abstract}

Keywords: Tinnitus; Onabotulinumtoxin A; myofascial pain; temporalis muscle; somatic tinnitus; migraine 


\section{INTRODUCTION}

Since the 1980 report of a therapeutic use for botulinum toxin, there has been a growing list of medical conditions benefited by its use 1 . Blepharospasm led serendipidously to its use for frown lines, which led serendipidously to its use for migraine ${ }^{2,3}$. In 2017 our trial of botulinum toxin type $A$ injections (OnabotulinumtoxinA, OnaA) for Chronic Migraine (CM) was reported ${ }^{4}$. Herein we report the trial's effect upon these same subjects' tinnitus, a serendipidous finding.

\section{MATERIALS AND METHODS}

57 patients ( $14 \mathrm{M}, 43 \mathrm{~F}$ ), who (i) fulfilled the international criteria for $\mathrm{CM}$ and (ii) were refractory to pharmacologic therapy, were treated with OnaA head and neck injections. In brief using both the location of the muscles with myofascial trigger points (MTrPs) and the distribution of the headaches, our "follow-the-pain" technique employed an adaptive procedure to decide which muscles to inject. All injections were bilateral as follows: if headaches were frontotemporal, then temporalis ( $30 \mathrm{U}$ per muscle) and corrugator (10 U); if cervico-occipital with trapezius MTrPs, trapezius ( $40 \mathrm{U})$; if both frontotemporal headaches and neck pain, all six muscles. Patients continued to use their prophylactic and abortive medications throughout. Because the headache benefit from OnaA typically persists about 3 months, injections were provided about every 3 months. To classify someone as a CM OnaA responder the number of headache days must decrease by $>50 \%$ from pre-treatment for two consecutive sets of injections. Prior to OnaA, subjects were not questioned about tinnitus. However some patients reported tinnitus improvement during the study. At that point going forward (a) new participants were questioned about tinnitus and (b) enrolled subjects were retrospectively questioned. If prior chronic tinnitus was reported, the subject underwent (a) audiometry, (b) an otorhinolaryngology evaluation and (c) questioning about the tinnitus' quality, loudness, localization, time course, precipitating and modifying factors, and relationship to their migraine.

\section{RESULTS}

Five women reported chronic tinnitus (Table 1); all were from the 41 OnaA CM responders. None had cervical MTrPs or pain, bruxism, jaw pain, temporomandibular dysfunction, otological or audiometric abnormalities. MTrPs in all five were in the temporalis and corrugator muscles, where OnaA was injected bilaterally, following our protocol.
Tinnitus began up to 33 years after migraine onset (mean: 20.4 years.), and 0 to 8 years after CM onset (mean: 2.8 years). Two subjects reported their tinnitus began at about the same time as their CM. Tinnitus was described as constant, non-lateralized, non-pulsatile, buzzing, and whistling or "as if someone breathed near my ear." Two described their tinnitus as louder to the side of their headaches. During about $75 \%$ of migraine attacks tinnitus loudness increased. All five patients described their tinnitus as disabling; two reported difficulty hearing during migraine attacks, which they attributed to the intensity of their tinnitus.

Within two to three weeks of the injections, tinnitus disappeared in two patients and in the other three their estimated loudness was reduced by more than $70 \%$. The tinnitus of patient $F / 58$ had been present for 10 years and was permanently abolished (now more than 5 years) with her first injections. It has not returned even though her headaches returned after about three months and required repeat injections. For the other four the tinnitus benefit persisted as long as the effect on CM (usually 3 to 4 months).

These patients initially were injected into bilateral corrugator and temporalis muscles. To investigate which muscle(s) was responsible for quieting tinnitus, each of the four subjects, whose tinnitus transiently responded, had only bilateral temporalis muscles injected at one of the routine repeat injections. It had the same effect upon the tinnitus (and the headaches) as when both corrugator and temporalis muscles were injected. We conclude that bilateral temporalis muscle injections only are sufficient to suppress their tinnitus (and headaches).

\section{DISCUSSION}

All patients with $\mathrm{CM}$-associated tinnitus had a major benefit to their tinnitus (and $\mathrm{CM}$ ) from temporalis muscle injections. In one case tinnitus of 10 years' duration was abolished permanently. In a second tinnitus was abolished repeatedly for about 3 months but recurred with the headaches. In the others tinnitus loudness was reduced by more than $70 \%$ repeatedly for about 3 months but recurred with the headaches. Just as occurred for the serendipitous findings that OnaA can treat frown lines and headache, our serendipitous finding for tinnitus now needs to be investigated with well-designed studies.

Because this study was designed to evaluate the response of $\mathrm{CM}$ to OnaA and not tinnitus, there is (a) a

Table 1: Tinnitus subjects' characteristics.

\begin{tabular}{ccccccccc}
\hline \multicolumn{7}{c}{ Onset age of } & \multicolumn{7}{c}{ Predominant Location } \\
\hline Sex/Age & Migraine & CM & Tinnitus & Migraine & Tinnitus & MTrPs & $\begin{array}{c}\text { Degree tinnitus } \\
\text { suppressed (\%) }\end{array}$ & suppression \\
F/58 & 15 & 48 & 48 & FT, R $>$ L & Bilateral R $>$ L & T & 100 & 5 years \\
F/46 & 12 & 35 & 43 & FT & Bilateral & T, C & 100 & 3 mos \\
F/63 & 20 & 45 & 45 & FT & Bilateral & T & 90 & 3 mos \\
F/55 & 15 & 22 & 25 & FT, R $>$ L & Bilateral R $>$ L & T, C & 80 & 3 mos \\
F/68 & 63 & 63 & 66 & FT & Bilateral & T & 70 & 3 mos
\end{tabular}

CM: chronic migraine, FT: frontotemporal,T: temporalis,C: corrugator,R: right,L: left, MTrPs: myofascial trigger points 
lack of rigor in the reporting of the subjects' tinnitus and (b) possibly recall bias. This raises the concern that tinnitus improvement is related to an overall improvement in well-being from headache improvement. However, wellbeing improvement would not account for the permanent abolishment of ten-year tinnitus, nor the tinnitus, which vanished repeatedly with each treatment.

Our study adds a novel dimension to the previous OnaA study 5 . The tinnitus of two of our subjects was abolished completely, while the prior study found no such subjects. On the other hand the prior study found a highly significant benefit $(p<0.001)$ for loudness perception. It was double blind placebo controlled, but did not target MTrPs. Unlike our study many had hearing loss and Meniere's syndrome. Also it used about half the total dose of our study, was subcutaneous, unilateral and into the periauricular region, while our injections were bilateral and intramuscular to the temporalis muscle. Nonetheless this well-designed study, like our incidental findings shows a significant benefit of OnaA for tinnitus. Taken together these studies support the need for more such studies investigating in particular the optimum targets and whether OnaA can significantly attenuate tinnitus of those without headache.

The question arises could decreased use of abortive medications from headache improvement account for tinnitus improvement, since (a) the headaches and tinnitus responses to OnaA were very similar and (b) abortive medications were used as needed. The major medication classes used as abortives are triptans and NSAIDs. While triptans are not associated with tinnitus, NSAIDs at high dosages can be. However case F/58 cannot be accounted for by a change in medication use, since her tinnitus was permanently abolished but her headaches were not. On the other hand this possibility cannot be ruled out for the other four cases. Future studies will settle this issue.

These results showing a close relationship between alleviation of both CM and tinnitus with OnaA are not surprising, since relief of head and neck myofascial dysfunction can alleviate both. For over 40 years it has been known that treating head and neck myofascial dysfunction can alleviate $\mathrm{CM}^{6}$. Similarly for over 20 years it has been known that treatment of head and neck myofascial disorders can abolish and or attenuate tinnitus ${ }^{7-10}$.

A second common feature of $\mathrm{CM}$ and tinnitus is that they share a close relationship to the Trigeminal-CervicalComplex (TCC) of the caudal brainstem/rostral spinal cord. The TCC is a "key relay center and major site of action" in migraine ${ }^{11}$. Similarly somatic tinnitus is closely related to the TCC and the tinnitus properties of our five $\mathrm{CM}$ subjects are consistent with the diagnosis of somatic (craniocervical) tinnitus. The following features of our subjects' tinnitus strongly support the categorization of their tinnitus as somatic tinnitus, i.e. head and neck myofascial etiology for their tinnitus. Their audiometry was normal, myofascial dysfunction (MTrPs) was present (in the temporalis muscles) and their tinnitus responded to intervention involving the craniocervical soft tissues ${ }^{12}$. Multiple lines of evidence support the concept that somatic tinnitus results from net disinhibition of the auditory CNS arising from the afferent sensory inputs of the Vth, Vllth, IXth, and Xth cranial nerves along with the $\mathrm{C} 1, \mathrm{C} 2$ and $\mathrm{C} 3$ dorsal spinal roots. These sensory afferents all converge upon a region of the lower medulla, the medullary somatosensory nucleus, a subunit of the TCC, from which activation of the CNS auditory perceptual machinery responsible for tinnitus emanates (Figure 1) ${ }^{9,12}$.

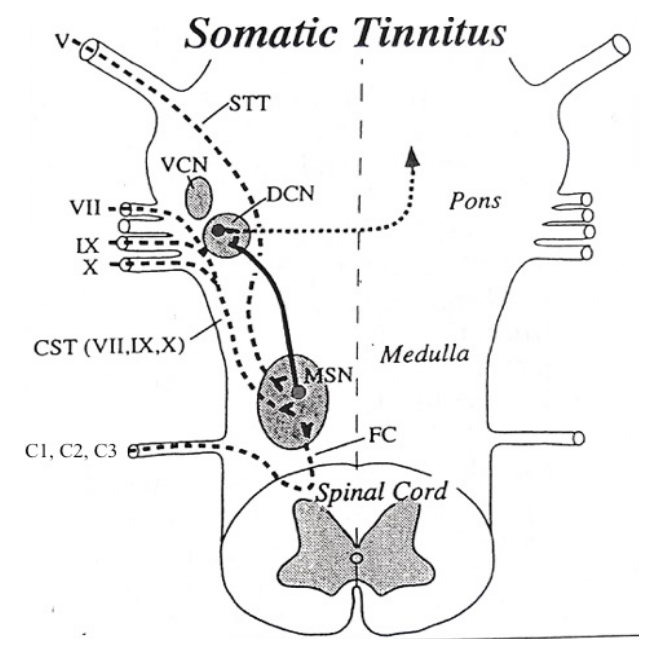

Figure 1: Schematic diagram of brainstem and upper cervical spinal cord, depicting the anatomic basis for somatic tinnitus. Tinnitus is caused by increased activity from the output of the dorsal cochlear nucleus (DCN) (curved arrow), which then projects to other centers and eventually leads to activation of the auditory perceptual machinery responsible for tinnitus. Sensory inputs (shown as long-dashed lines) from (1) the face (via the trigeminal nerve [V] and the spinal trigeminal tract [STT]), (2) the external and middle ears (via the common spinal tract of the facial, glossopharyngeal, and vagus nerves (CST[VII, IX, XI]), and (3) the neck (via the $\mathrm{C} 1, \mathrm{C} 2$, and $\mathrm{C} 3$ dorsal roots and the fasciculus cuneatus [FC]) converge to a common region of the lower medulla, the medullary somatosensory nuclei (MSN) a part of the TCC, from which fibers project to the ipsilateral DCN (solid line). When modulation of the activity in the MSN to DCN pathway results in disinhibition of DCN, tinnitus can occur. VCN, ventral cochlear nucleus. 
OnaA is known to have peripheral and central effects ${ }^{13}$. Peripherally it affects efferents by blocking acetylcholine release at the neuromuscular junction, and afferents by inhibiting the release of neurotransmitters that activate sensory neurons, thereby reducing myofascial pain. Centrally animal models suggest that its effect involves the TCC either indirectly (by reducing myofascial peripheral inputs to the CNS) or directly through retrograde axonal transport. The similar time course of tinnitus and CM attenuation (for four of our five subjects) suggests that, wherever OnaA is having its effect, it is the same for both conditions and it involves the structures they share in common.

\section{CONCLUSION}

OnaA injections when used for CM in the "follow the pain" mode can permanently abolish $\mathrm{CM}$ associated tinnitus in some and in others can attenuate it by more than $70 \%$ for about three months.

\section{CONFLICT OF INTEREST}

The authors declares no conflict of interest.

\section{REFERENCES}

1. Scott $A B$. Botulinum Toxin Injection into Extraocular Muscles as an Alternative to Strabismus Surgery. Ophthalmology. 1980;64:35127-60.

2. Binder WJ, Blitzer A, Brin MF. Treatment of hyperfunctional lines of the face with Botulinum toxin A. Dermatologic Surg.1998;26:1-6.

3. Carruthers JDA, Carruthers JA. Treatment of Glabellar Frown Lines. J Dermatol Surg Oncol. 1992;12:10-26.

4. Ranoux D, Martine G, Espagne-Dubreuilh G, Amilhaud-Bor$\operatorname{dier}$ M, Caire F, Magy L et al. Injections in chronic migraine, targeted to sites of pericranial myofascial pain: an observational, open label, real-life cohort study. J Headache Pain. 2017; 14:781-6.
5. Stidham KR, Solomon PH, Roberson JB. Evaluation of botulinum toxin in treatment of tinnitus. Otolaryngol Head Neck Surg. 2005;2;112-16.

6. Tfelt-Hansen P, Lous I, Olesen J. Prevalence and Significance of Muscle Tenderness during Common Migraine Attacks. Headache J Head Face Pain. 1981;24;130-6.

7. Eriksson-Mangold M, Gustafsson S, Axelsson A. Tinnitus and trigger points: A randomized cross-over study. In: Proceedings of the Fifth International Tinnitus Seminar. 1996;20;114-16.

8. Gritsenko K, Caldwell W, Shaparin N, Vydyanathan A, Kosharskyy B. Resolution of long Standing tinnitus following radiofrequency ablation of $\mathrm{C} 2-\mathrm{C} 3$ medial branches - A case report. Pain Physician. 2014;1:101-10.

9. Levine RA. Somatic (craniocervical) tinnitus and the dorsal cochlear nucleus hypothesis. Am J Otolaryngol Head Neck Med Surg. 1999;20:351-62.

10. Goadsby PJ, Holland PR, Martins-Oliveira M, Hoffmann J, Schankin C, Akerman S. Pathophysiology of migraine: A disorder of sensory processing. Physiol Rev. 2017;2:214-18.

11. Levine RA. Somatic tinnitus. In: Snow JB, edition. Tinnitus: Theory and Management. BC Decker Inc. 2004;1:108-124.

12. Matak I, Bolcskei K, Bach-Rojecky L, Helyes Z. Mechanisms of botulinum toxin type $A$ action on pain. Toxins (Basel). 2019;2;1-6.

13. Manuel Muñoz-Lora VR, Abdalla HB, Del Bel Cury AA, Clemente-Napimoga JT. Modulatory effect of botulinum toxin type $A$ on the microglial P2X7/CatS/FKN activated-pathway in antigen-induced arthritis of the temporomandibular joint of rats. Toxicon. 2020;187:116-121. 